Our thanks to all those who have helped with this issue of Bioanalysis. Listed below are authors, referees and others who have kindly given their time, effort and expertise; their generosity has helped establish this publication.

$\begin{array}{llll}\text { Ahnoff M } & \text { Ewles M } & \text { Koupaei-Abyazani M } & \text { Price W } \\ \text { Aubry A } & \text { French D } & \text { Ledvina A } & \text { Pucci V } \\ \text { Bansal S } & \text { Furtado M } & \text { Li F } & \text { Shah K } \\ \text { Blackburn M } & \text { Garofolo F } & \text { Liu A } & \text { Solich P } \\ \text { Brummel C } & \text { Gray N } & \text { Lucena R } & \text { Termopoli V } \\ \text { Brus T } & \text { Guo X } & \text { Mess J } & \text { van de Merbel N } \\ \text { Campbell L } & \text { Havlíková L } & \text { Mulvana D } & \text { van Eeckhaut A } \\ \text { Cappiello A } & \text { Ho S } & \text { Neville D } & \text { Vlcková H } \\ \text { Danaceau J } & \text { Ismaiel O } & \text { Nováková L } & \text { Yuan L } \\ \text { de Vries R } & \text { Jelvestam M } & \text { Olsson A } & \\ \text { DiFrancesco R } & \text { Ji Q } & \text { Pelzer M } & \\ \text { Doolittle D } & \text { Kagel J } & \text { Peoples M } & \end{array}$

\title{
INDEX OF ADVERTISERS
}

\begin{tabular}{lr} 
Company & Page \\
\hline ejournal collection & Inside front cover \\
European Bioanalysis Forum & $\mathrm{V}$ \\
Waters & VII \\
Bioanalysis Zone - get in the zone & VIII \\
Merck Millipore & $I X$ \\
Join the Bioanalysis Zone & $X$ \\
Publish your research with Bioanalysis & 2336 \\
Bioanalysis trial & 2340 \\
Advertise with Bioanalysis & 2358 \\
Subscribe to Bioanalysis & 2392 \\
Bioanalysis zone - have your say & 2408 \\
\hline
\end{tabular}

